\title{
Field study on economic impact of the working environments in non-temperate climates
}

\author{
R. M. Ahmed \\ The Bartlett School of Graduate Studies, University College London, UK
}

\begin{abstract}
During the past few decades and in the rapidly developing tropical climates of Asia-Pacific regions and the hot arid climates of the Arabian Peninsula, engineers have focused on creating comfortable indoor environments inductive to productivity in an aim to promote "sustainable development" with a resultant heavy reliance on HVACs for ventilation. Research on office performance has shown that occupants' exposures to inadequate ventilation rates and to elevated or very low temperatures attribute to a higher prevalence of employees' illnesscaused absence; which, in a sense, is reflected in their income. However, scare data is available regarding to which extent the working environment has affected employees' income particularly that the effect of temperature, thermal comfort and quality of air from the HVAC systems used in the Arabian Peninsula is less understood. This study is investigating the impact of the working environments on employees' economic status in non-temperate climates. A field study of thermal comfort was conducted in Saudi Arabia, during the fall of 2013, in which over 800 workers responded to a questionnaire while simultaneous physical measurements were taken. The results suggest that employees in Saudi Arabia became highly accustomed to air conditioners; perceiving temperature $22^{\circ} \mathrm{C}$ as the comfortable temperature compared to $25^{\circ} \mathrm{C}$. However, their productivity has been impaired and their absenteeism rates have doubled with an equivalent salary deduction of 1/8 of the typical payment per annum.

Keywords: field study, indoor temperature, indoor air quality (IAQ), nontemperate climates, working environments, employees' productivity, illnesscased absence, economic impact.
\end{abstract}




\section{Introduction}

When most people hear the word "economic", they always think of costs and the management of money. In particular, economic impact of the working environments suggests more than how better the working environments can be for improving the economic status of workers. In other words, the macroeconomic estimates of the nationwide financial gains that have been developed for cost-effective built environments are emphasizing that indoor environmental conditions of any working space substantially influence employees' health and performance. Without going deeply into theories of economics, it is enough to say that in any organisation, within the principles of health and safety management, costs paid or expected to be paid for workers in the case of accidents (including diseases as well) are attributable to the conditions of work, and not the performance of work [1]. Fisk et al. [2] have estimated a qualitative relationship between ventilation rates and sick leaves in offices, indicating that respiratory infections and illnesses leading to sick leaves are highly dependent on the effectiveness of ventilation, derived from a survey incorporating 100 US office buildings. In another study by Wargocki et al. [3], it was evident that, performance of work decrease by $1.1 \%$ for each $10 \%$ increase in percentage dissatisfied of air quality by workers. Moreover, a series of experiments have indicated the direct correlation between sick buildings' syndromes (SBS) and work performance (e.g. [4-6]). In addition, studies on SBS and temperature reported a linkage between high temperatures and higher prevalence of SBS symptoms (e.g. [7]). Furthermore, a number of studies have shown that elevated or very low indoor ambient temperatures impair performance (e.g. $[8,9])$. Thus, the inter-related relationship between physical environments of working spaces, employees' work performance illness-caused absence is evident. However, the effect of temperature on workers' productivity and the effect on illness-caused absence has received much less attention [9], although it has been proven that air temperature influences performance indirectly through its impact on prevalence of SBS symptoms or satisfaction with air quality $[10,11]$.

This is particularity more critical to the rapidly developing harsh desert climate countries of the Arabian Peninsula relying heavily on HVAC for ventilation as a mean by which engineers and architects provided occupants with "thermal comfort" where it was impossible to extract the heat from the uninsulated buildings by any other mean, given that the average temperature in the region reaches as high as $54^{\circ} \mathrm{C}$ in some places throughout 9 months of the year [12]. After the mid 70s, in response to the oil shocks, the path of the region has been transferred towards modernization and sustainable development. This phenomenon has led to huge energy consumption in the building stock. By looking at Saudi Arabia for instance, since it occupies about $80 \%$ of the region [13], the energy consumption of buildings from air conditioners in all sectors has reached $70 \%$ in 2011 [14]. 


\subsection{How indoor temperature of spaces is related to the mechanisms of communicable respiratory illness transmission?}

Empirical evidence has shown that respiratory illnesses are highly correlated to the quality of indoor environments, sick building symptoms and worker performance; with reference to air temperature, efficiency or rate of air filtration, the rate of ventilation (i.e. supply of outside air per occupant), and humidity which were found to be influencing the prevalence and period of viability of transmission of airborne infectious aerosols; including common colds, influenza, adenovirus infections, measles, and other common respiratory illnesses. As a result of reduced respiratory disease in the United States for instance, potential annual savings and productivity gains were estimated with around $\$ 6$ to $\$ 14$ billion [15].

This was found to be in line with the findings of Mourtzoukou and Falagas [16], who have linked the increased incidence and severity of respiratory tract infections to cold exposures. It was suggested that exposure to the cold, either through exposure to low environmental temperatures or during induced hypothermia, attribute to an increase in the risk of developing upper and lower respiratory tract infections, pointing out to the fact that; the longer the duration of exposure the higher the risk of infection. This has been based on the fact that, inhaled cold air, cooling of the body surface and cold stress induced by lowering the core body temperature cause pathophysiological responses such as vasoconstriction in the respiratory tract mucosa and suppression of immune responses, which are responsible for increased susceptibility to infections.

\section{Methods}

A field study was conducted in Jeddah during fall 2013 employing questionnaire surveys and simultaneous physical measurements of the thermal environment in two selected buildings. The selected buildings and experiment protocol are described below.

\subsection{The case study buildings}

The first case study building is a university building denoted as A. The second building is an office building denoted as B, and constructed in the same period as building A. The selected buildings are typical examples of reinforced concrete modern architecture structure in Saudi Arabia. Both buildings had no thermal insulation on the external walls. Building A comprised of 75 offices incorporating around 525 employees. Building B comprised of 53 offices incorporating around 370 employees. The HVAC system used in both buildings is a centralised constant air volume (CAV) system where offices' temperature and ventilation rates were controlled using a building management system (BMS). 


\subsection{Experiment protocol}

It is worth mentioning that before conducting the study in the selected buildings, 20 newly built randomly selected buildings were visited, from commercial and institutional sectors. This was in an aim to acquire information about the temperature and ventilation rates prevalent; acting as the primary criteria for selecting the case study buildings where it was crucial to select buildings with significantly different prevalent temperatures (known as baseline temperature) for a feasible comparison between the prevalence of SBS, attributed to illnesscaused absence rates and salary deduction over the whole year from the two selected buildings. It was equally important to select adequately ventilated buildings; with not less than 8 liters/sec/person, as per the recommendations of ASHRAE standards (the American Society of Heating, Refrigerating and Air Conditioning Engineers), in order to ensure that the only reason attributed to illness-cased absence was the employees' physiological response to thermal perception of discomfort and/or due to the effect of temperature on the prevalence of SBS but not ventilation rates. The required information have been attained via physical monitoring of indoor ambient temperature and air speed from air conditioners' return and supply diffusers. The measurement of the return air flow was deducted from the supply air flow to estimate the net air flow rate then divided by the number of people in the room to estimate the ventilation rate per liter/ sec./ person. The monitoring period lasted for 20 days (1 day/ building) during the occupancy hours. Also it was crucially important to choose the buildings which have centralized air conditioning with no individual thermostat in the offices in order to make sure that the employees were not changing the temperature during the time of exposure.

Hence, building A was selected with baseline temperature of $22^{\circ} \mathrm{C}$ and building $\mathrm{B}$ with baseline temperature of $25^{\circ} \mathrm{C}$ with a ventilation rate of 6 lit./sec./person in both buildings. The physical monitoring protocol was designed with independent variable of temperature, where the office temperature was set at $20,21,22,23,24$ and $25^{\circ} \mathrm{C}$, since the majority of studies have linked performance decrements to temperatures between $20^{\circ} \mathrm{C}$ and $25^{\circ} \mathrm{C}$, whereas the ventilation rate was kept constant throughout out the experiment at 6 lit./sec./person. Each exposure condition lasted for 1 week, for both buildings simultaneously. At the same time, subjective responses from the employees were collected in order to compare the reported thermal perception in the different exposure conditions. In addition, outdoor temperature and relative humidity were monitored. Indoor air temperature was measured using $\mathrm{HOBO} \mathrm{U} 12\left( \pm 0.2^{\circ} \mathrm{C}\right.$ and $\pm 2 \%$ ), air velocity was measured using Testo 4005634170417 Large Vane Anemometer $( \pm 1.5 \%$ of reading) and outdoor temperature and relative humidity were measured using HOBO UX1000-003 $\left( \pm 0.2^{\circ} \mathrm{C}\right.$ and $\left.\pm 3.5 \%\right)$.

Employees' thermal comfort was estimated using a questionnaire form employing questions on employees' thermal sensation votes (TSV) which were casted on the ASHRAE/ISO seven-point thermal sensation scale, defined as: Hot (3), warm (2), mild warm (1), neutral (0), mild cool (-1), cool (-2) and cold (-3). The questionnaires also included questions regarding the prevalence of sick 
building syndromes (SBS); like watery eyes, dry throats and runny nose, during the experiment weeks and during the past year, in addition to a question about the effect of thermal perception on the frequency of illness-caused absence attributed to human body physiological response to the cold exposure; like common cold, influenza, or respiratory illnesses. Also, a question on the average absence rate during the past year with the subsequent salary deduction rate was included. Moreover, it was important to ask about the use of air conditioning trend at home and the temperature setting in order to understand to which extent the people got acclimatized to the cold. A version of the survey questionnaire is provided in Appendix A. Nevertheless, a brief interview with the accounting manager was conducted in both buildings, asking about the policy adopted for sick leaves and salary deduction. This was to co-relate the employees' responses from the questionnaires and the estimated salary decrement per year. Likewise, a brief interview with the personnel responsible for temperature control was conducted, asking about the criteria based on which the baseline temperature was set.

For data analysis, employees' self-reported thermal sensation votes during the weeks of exposure were plotted against the 6 temperatures of the experiment using Microsoft excel. Similarly, employees' self-reported frequency of using air conditioners at home was compared from both buildings and charts were drawn on excel sheets. Employees' SBS self-reported subjective responses and the reported illnesses associated with physiological responses to the cold were compared to the prevalence of absence and salary deduction from both buildings, where charts were drawn on excel sheets as well. The independent samples t-test was used to check the significance of exposing employees to comfortable mild cool office temperature compared to comfortable mild warm temperature on the prevalence of illness-caused absence/ month between the two groups (A and B). A two tailed t-test was computed with type 3 where an un-equal variance was assumed between the two groups (Leven's Test).

\section{Results and discussion}

The results of the thermal sensation votes from employees in both during each of the exposure condition are provided below, with reference to the baseline temperature in each building.

When employees' self-reported frequency of using air conditioners at home was compared from both buildings, it was found that employees from building A use air conditioning at home for almost 24 hours per day all over the year even during winter at temperature $\approx 21-22^{\circ} \mathrm{C}$. Employees from building $\mathrm{B}$ use air conditioning at home for less than 24 hours per day $(\approx 18$ hours) for 9 months (all over the year except during winter) at temperature $\approx 24-25^{\circ} \mathrm{C}$. Accordingly, one can suggest that the low temperature set point preferred by the occupants in building A is mostly attributed to the effect of habituation which is known to have a direct correlation with people's psychological expectations. 


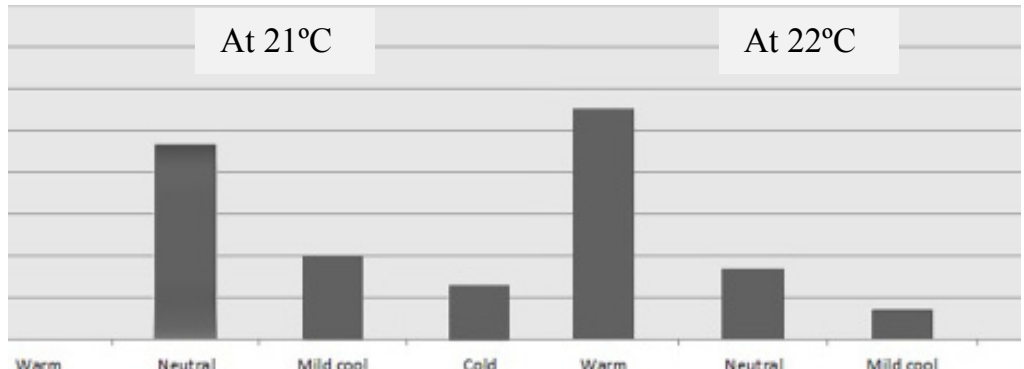

Figure 1: The baseline temperature in building A (see Appendix B for details).

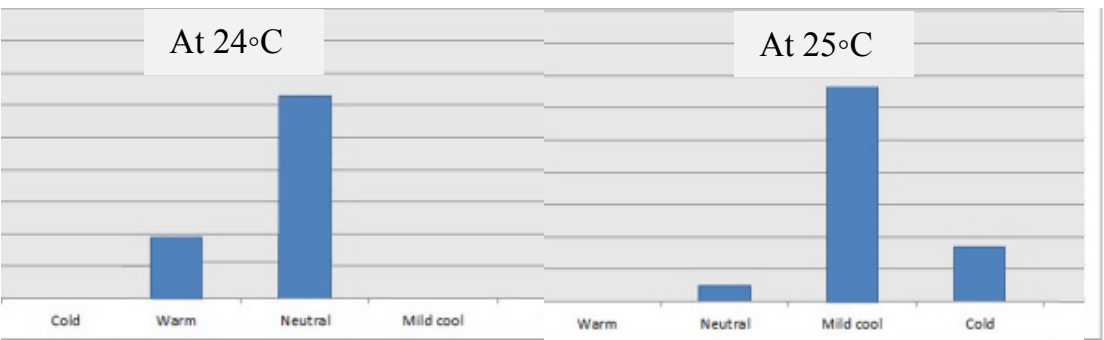

Figure 2: The baseline temperature in building B (see Appendix B for details).

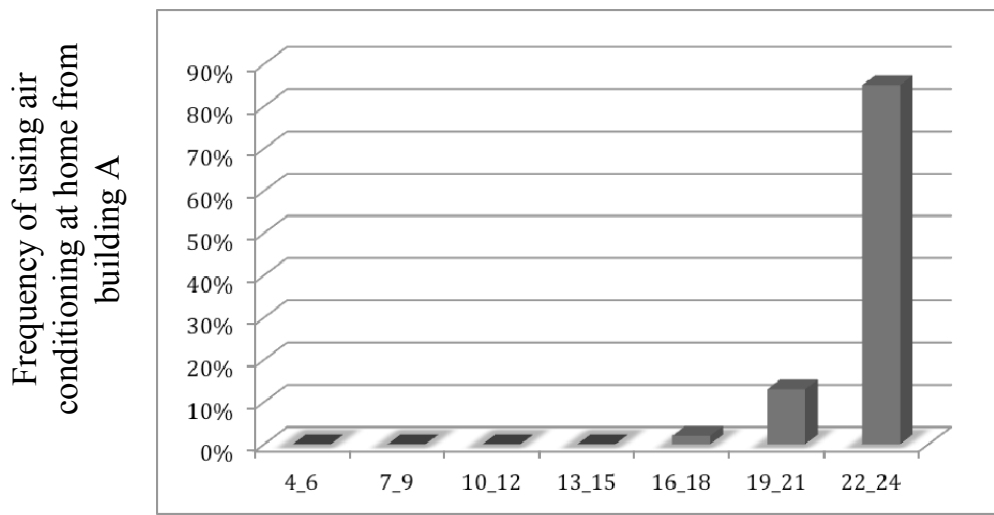

Number of hours per day

Figure 3: The frequency of using air conditioning at home from building A during summer and winter. 

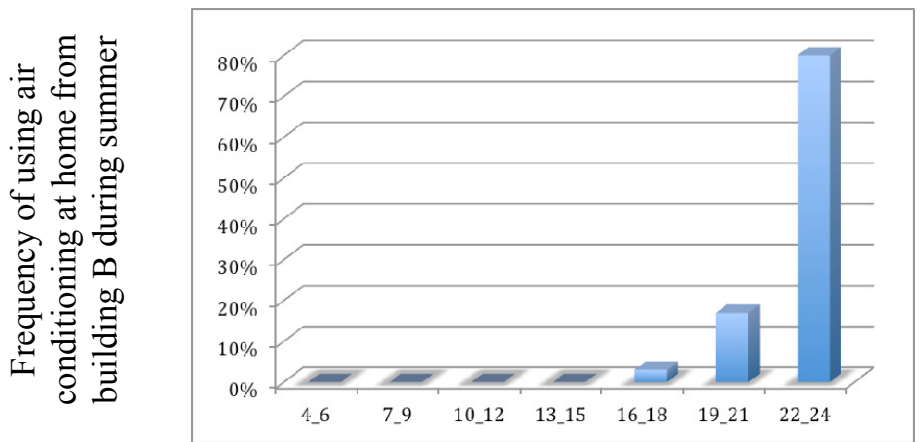

Number of hours per day
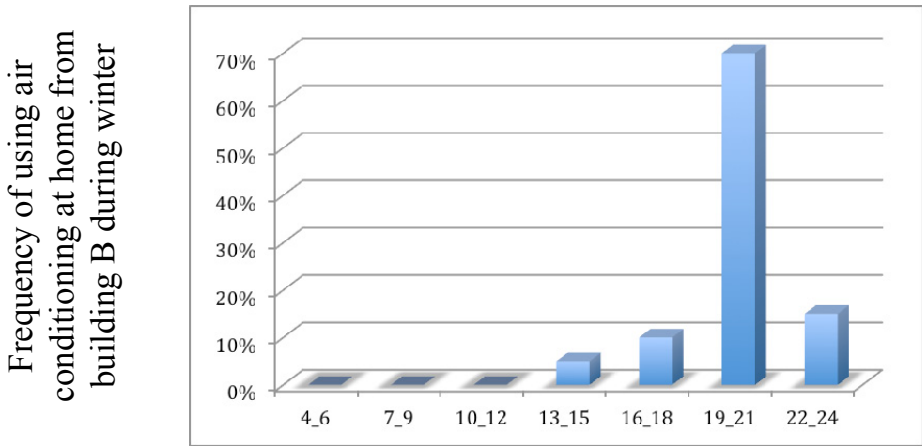

Number of hours per day

Figure 4: The frequency of using air conditioning at home from building B during summer and winter.

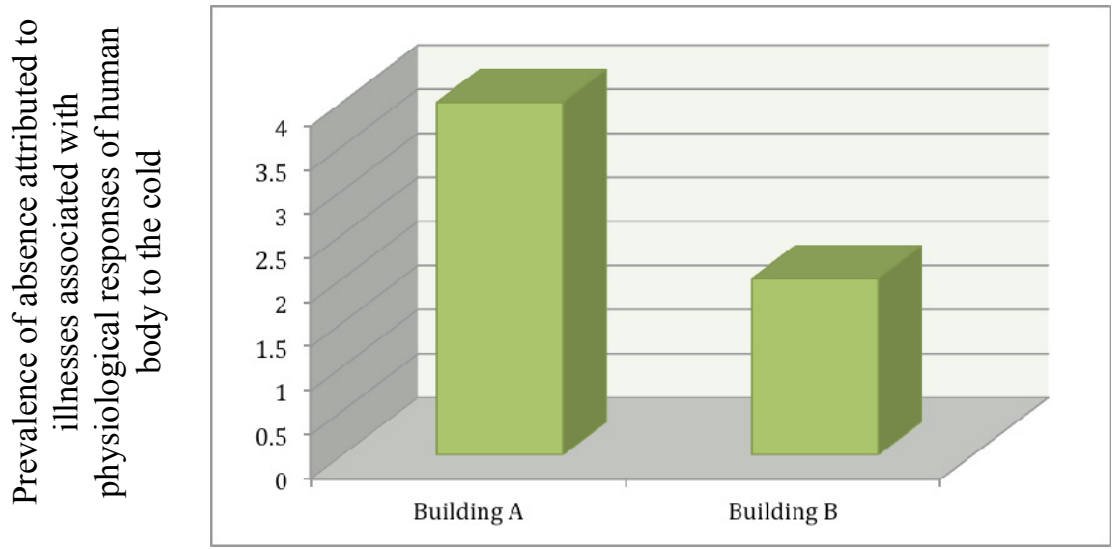

Figure 5: Prevalence of absence attributed to illnesses associated with physiological responses of human body to the cold from both buildings. 
For understanding how this affected employees' performance and absence at work, the self-reported sick building syndromes during the previous year of from both buildings were then compared to the employees' overall thermal perception and the prevalence of illness-caused absence. It was found that the reported absence due to illnesses attributed to physiological responses of human body to the cold (like cold, headache and runny nose) are the ones which led to salary deduction since they were associated with acute illnesses and subsequent absence exceeding the 5 days excused absence allowed per year as per the sick leave policy adopted in the organisations (information provided based on the interviews conducted with the accounting managers).
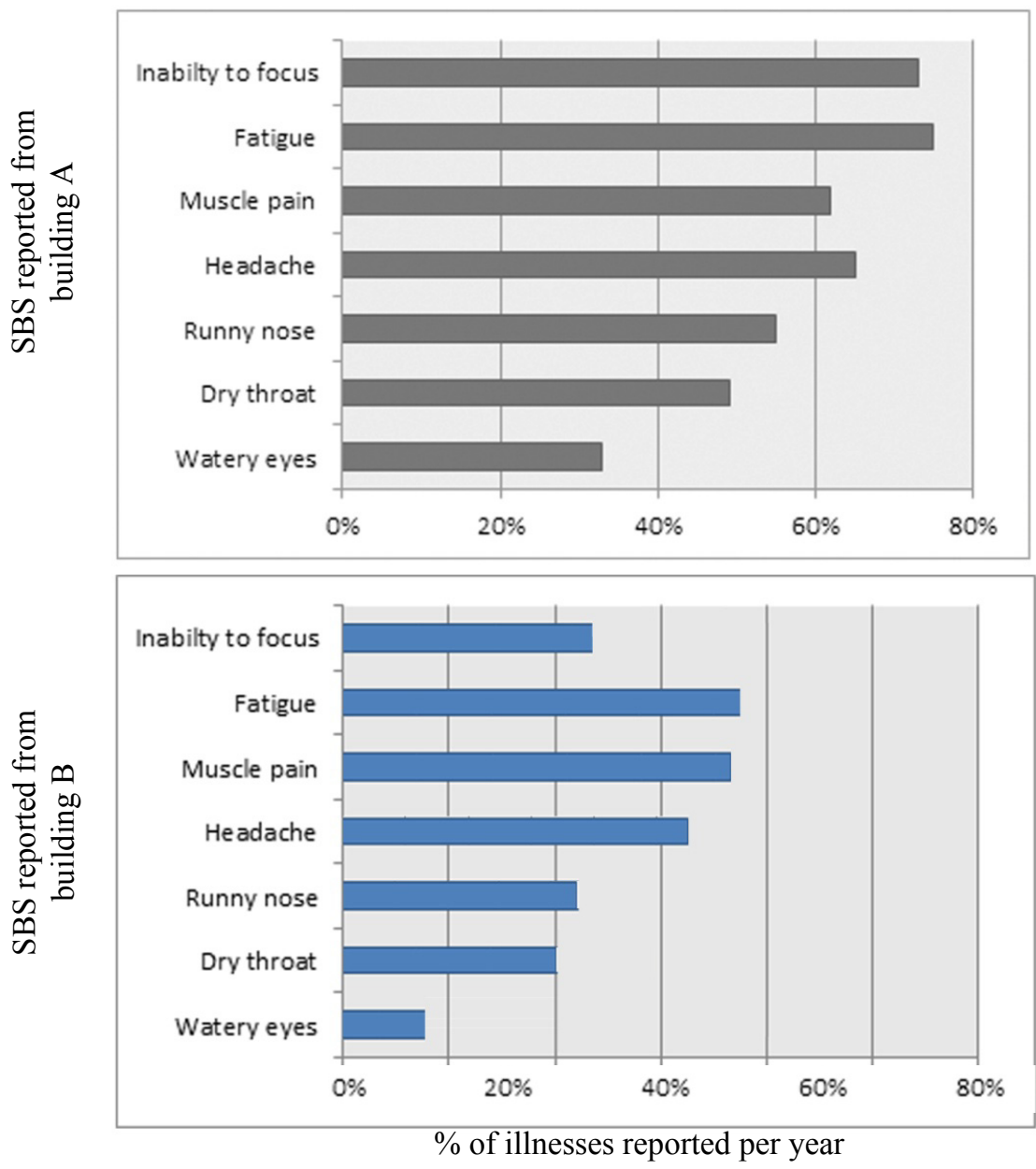

Figure 6: $\quad$ SBS reported from buildings A and B.

Most importantly, it was found that in building A, there is a sample of $15 \%$ employees perceiving the baseline temperature $22^{\circ} \mathrm{C}$ as cold. Corresponding 
$40 \%$ higher SBS have been reported compared to the "acclimatized" colleagues with a resultant of acute illness (mostly respiratory) which caused a significant increase in their prevalence of illness-caused absence. Nevertheless, the comparison of self-reported prevalence of SBS from employees in both buildings with specific attention to the symptoms of inability to focus, fatigue and headache (evident to impair performance directly [17]) were significantly less in building $\mathrm{B}$ with baseline temperature $25^{\circ} \mathrm{C}$ compared to building A with baseline temperature $22^{\circ} \mathrm{C}$ which implies a high correlation of SBS with cold prolonged exposure in the hot countries although cold sensation was preferred by the majority of occupants.

The $\mathrm{t}$ test results indicated significance difference $(\mathrm{P}=0.0001)$, i.e. a significant effect was found (mean illness-caused absence prevalence/month $\mathrm{A}=3.44$, mean illness-caused absence prevalence/ month $\mathrm{B}=1.77$ ). The same method was applied to determine the effect on the difference between employees' salary deduction per month between the two groups. $\mathrm{P}=0.005$, i.e. again a significant difference was found. Based on a comparison between the mean salary deduction/month of the two groups, the mean salary deduction/year was estimated of an average $0.125 \%$ per year from group A compared to an average $0.125 \%$ per year from group B. Therefore $1 / 8$ of the annual income is found to be an average of salary decrement caused due to illness-caused absence attributed to physiological responses of human body to the cold.

\section{Conclusions}

It was not surprising to find scholars and researchers striving to defeat the sustainable development challenges encountered in the Arabian Peninsula due to the arid nature of the region regardless to costs; after energy has become cheap and affordable. Unfortunately, this phenomenon has led to a huge energy consumption from air conditioning in the building stock; whereas at the first place, the concept of sustainable development has been based on the attempt to combine the growing concerns of a specific society about a range of environmental issues with their socio-economic issues, which determined considerations of the society's social norms and behaviors not only economic status.

It could be therefore suggested that, although the new building technologies employed in the rapidly developing country of Saudi Arabia to achieve modernity, high quality of living, and comfortable buildings with the intention of achieving sustainable development. However, in return, it was found that neutral thermal sensation due to getting highly accustomed to the cold exposures was not able change humans' thermoregulatory system and physiological response to cold stress easily; although it was capable of altering occupants' behaviors and expectations, with the consequence of higher prevalence of illness-caused absence reflected on the employees' salary deduction. An equivalent salary deduction of $1 / 8$ of the typical payment per annum has been estimated.

Overall, the decreased prevalence of illness-caused absenteeism rates estimated from elevating offices' ambient temperature from $22^{\circ} \mathrm{C}$ to $25^{\circ} \mathrm{C}$ could 
serve as a strong stimulus for energy efficiency measures that simultaneously improve the personal income of employees.

\section{Appendix A}

Q1. Today's date: $($ Day/Month/Year $) \ldots / \ldots$ Q2. Age:

Q3. For how long have you been working in this institution:

Q4. Generally, how do you perceive the offices' temperature in this building? (If your thermal comfort sensation level is varying from time to time, try to give an average rating)

$\begin{array}{ccccccc}-3 & -2 & -1 & 0 & 1 & 2 & 3 \\ \text { Cold } & & & \text { neutral } & & & \text { Hot }\end{array}$

Q5. Right now, how you feel about the current office temperature? Try to give an average rating
$-3$
$-2$
$-1$
0
1
2
3
Cold
neutral
Hot

5.1 Would you like to change it?

Yes

No

Q6. Generally all over the year, due to the sensation of cold while you're in the building, have you had any of the following syndromes?
Common cold
Dry throats
Runny nose
Headache Inability to focus Influenza Respiratory illnesses
Fatigue Muscle pain

Have any of these symptoms led to absence from work?

Yes No

Was there any salary deduction due to this illness-caused absence?

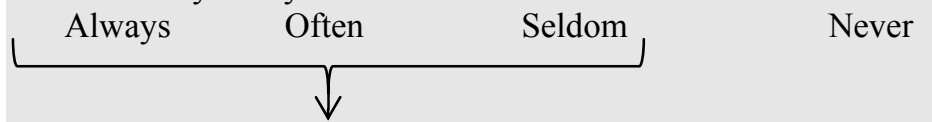

Can you give an estimate percentage of deduction per month?

Q7. At home, how often you use air conditioners?

During summer:

4-6 hrs 7-9 hrs 10-12 hrs 13-15 hrs 16-18 hrs

19-21 hrs 22-24 hrs

What is the temperature setting? ......... ${ }^{\circ} \mathrm{C}$ (try to give an average value)

During winter:
4-6 hrs
7-9 hrs
$10-12 \mathrm{hrs}$
13-15 hrs
16-18 hrs
19-21 hrs
22-24 hrs
What is the temperature setting? ${ }^{\circ} \mathrm{C}$ (try to give an average value)

END - THANK YOU FOR YOUR COOPERATION 


\section{Appendix B}

\section{Building A}
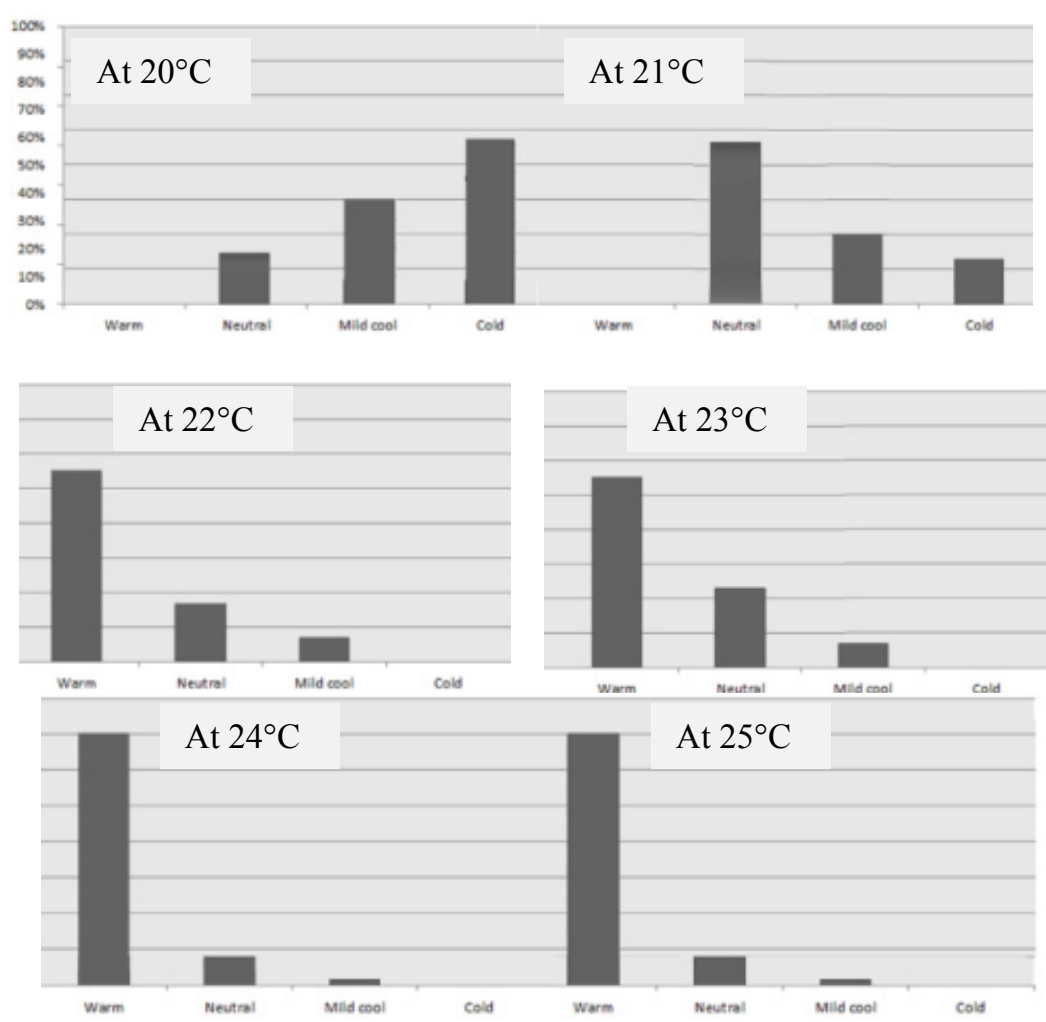

\section{Building B}

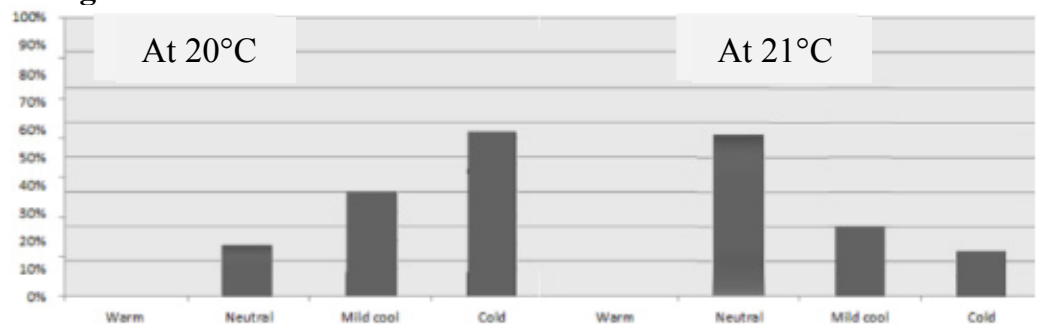



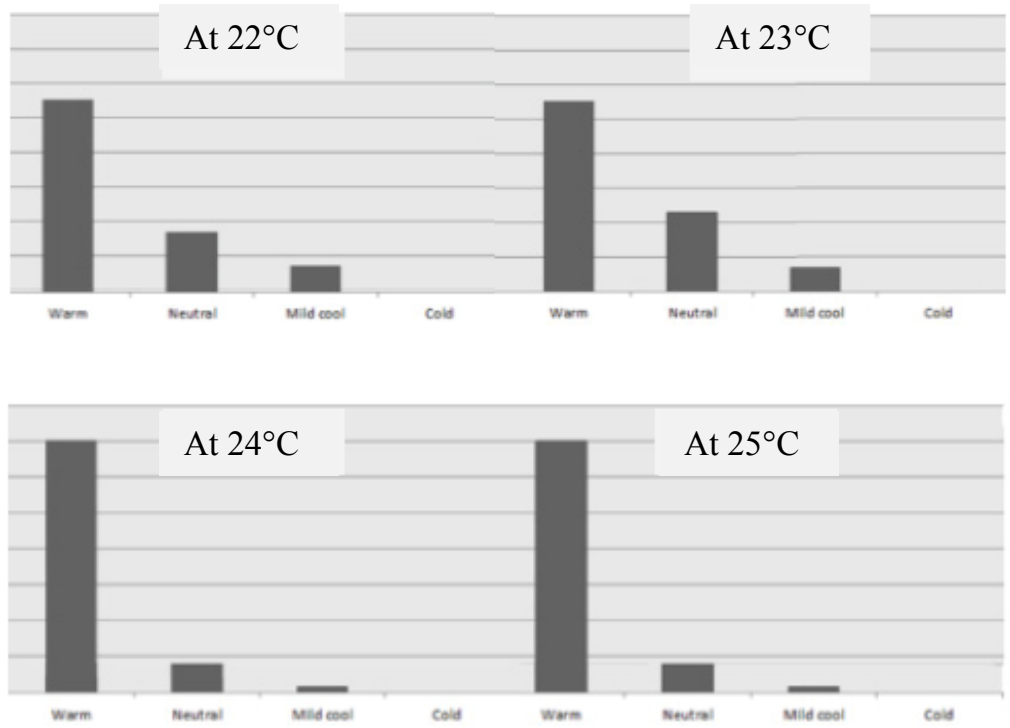

\section{References}

[1] Dorman P. The Economics of Safety, Health, and Well-Being at Work: An Overview. Online. http://oit.org/wcmsp5/groups/public/ed_protect/— protrav/—_afework/documents/publication/wcms_110382 .pdf

[2] Fisk W.J., Seppannen O., Faulkner D., Huang J. Economizer system cost effectiveness: accounting for the influence of ventilation rate on sick leave. Proceedings of ISIAQ $7^{\text {th }}$ International Conference Healthy Buildings 2003. December 7-12, Singapore, (3), pp. 361-367.

[3] Wargocki P., Wyon D.P., Sundell J., Clausen G. and Fanger P.O. The effects of outdoor air supply rate in an office on perceived air quality, Sick Building Syndrome (SBS) symptoms and productivity. Indoor Air, (10), pp. 222-236, 2000.

[4] Wargocki P., Wyon D.P., Baik, Y.K., Clausen G. and Fanger P.O. Perceived air quality, Sick Building Syndrome (SBS) symptoms and productivity in an office with two different pollution loads. Indoor Air, (9), pp. 165-179, 1999.

[5] Lagencranz L., Wistrand M., Willen U., Wargocki P., Witterseh T., and Sundell J. Negative impact of air pollution on productivity: Previous Danish findings repeated in new Swedish test. Proceedings of the Healthy Buildings 2000 Conference, (2), pp. 653-58.

[6] Bakó-Biró Z., Wargocki P., Weschler C. and Fanger P.O. Effects of pollution from personal computers on perceived air quality, SBS symptoms and productivity in offices. Indoor Air. (14), pp. 178-187, 2004. 
[7] Mendell, M.J., Fisk W.J., Petersen M.R., Hines C.J., Dong M., Faulkner D., Deddens J.A., Ruder A.M., Sullivan D., and Boeniger M.F. Indoor particles and symptoms among office workers: Result from a double-blind cross-over study. Epidemiology, (13), pp. 296-304, 2002.

[8] Seppänen, O., Fisk W.J., Faulkner D. Cost benefit analysis of the nighttime ventilative cooling. In: Proceedings of the Healthy Buildings 2003 Conference. Singapore, (3), pp. 394-399, 2003.

[9] Seppänen, O., Fisk W.J. Some quantitative relationships between indoor environmental quality and work performance or health. $H V A C \& R$ Research. 12(4), pp. 957-973, 2006.

[10] Seppänen O.A., Fisk W.J., Mendell M.J., Association of ventilation rates and $\mathrm{CO}_{2}$-concentrations with health and other responses in commercial and institutional buildings. Indoor Air, (9), pp. 252-74, 1999.

[11] Fang L., Wyon D.P., Clausen G., Fanger P.O., Impact of indoor air temperature and humidity in an office on perceived air quality, SBS symptoms and performance. Indoor Air, (14), pp. 74-81, 2004.

[12] Arabian Desert, Online. http://www.britannica.com/EBchecked/topic /31610/Arabian-Desert/47941/Climate

[13] Stokes J. Encyclopedia of the People of Africa and the Middle East. (1), pp. 605, 2009.

[14] Bachellerie I.J. Renewable energy on the GCC countries. Gulf Research Centre, 2012.

[15] Fisk W.J. Estimates of potential nationwide productivity and health benefits from better indoor environments: an update. In: Spengler, J. Sammet J. and McCarthy, J. eds. Indoor Air Quality Handbook, McGraw Hill, 2001.

[16] Mourtzoukou E.G., and Falagas M.E. Exposure to cold and respiratory tract infections. In: Int J Tuberc Lung Dis. 11(9), pp. 938-43, 2007.

[17] Reid M. Fatigue risk management system, Queensland, 2009. 